\title{
Tunneling spectroscopy on semiconductors with a low surface state density
}

\author{
Ch. Sommerhalter, Th. W. Matthes, ${ }^{\text {a) }}$ J. Boneberg, and P. Leiderer \\ Fakultät für Physik, Universität Konstanz, Postfach 5560, D-78434 Konstanz, Germany \\ M. Ch. Lux-Steiner \\ Hahn-Meitner-Institut, Glienicker Str. 100, D-14109 Berlin, Germany
}

(Received 3 January 1997; accepted 15 August 1997)

\begin{abstract}
A detailed study of tunneling spectroscopy concerning semiconductors with a low surface state density is presented. For this purpose, $I-V$ curves under dark conditions and under illumination were measured on the (0001) van der Waals surface of a $p$-type $\mathrm{WS}_{2}$ single crystal, which is known to be free of intrinsic surface states. The measurements are interpreted by an analytical one-dimensional metal-insulator-semiconductor model, which shows that the presence of the finite tunneling current has to be considered in the calculation of the tip-induced bandbending. Rectification of the dark $I-V$ curves is explained by the absence of an inversion layer at the semiconductor surface. In contrast, the $I-V$ curves measured for different light intensities and tip-sample separations indicate the existence of an optically induced inversion layer. Since no surface recombination needs to be considered to model these spectra, we conclude that bulk recombination, diffusion and direct tunneling of photogenerated minority charge carriers are the dominant processes for semiconductors with a low density of surface states. In contrast to the standard interpretation of tunneling spectroscopy, which can be applied to semiconductors with a high surface state density, our results clearly show that in this case the normalized differential conductivity $(d I / d U) /(I / U)$ cannot be used to determine the energetic distribution of the local surface state density.
\end{abstract}

\section{INTRODUCTION}

Scanning tunneling spectroscopy (STS) methods have been intensively used to study the electronic properties of semiconductor surfaces on a nm scale. ${ }^{1-4}$ For semiconductors with a high surface state density in the forbidden band gap, it is usually assumed that the presence of the tunneling tip does not influence the electronic properties of the semiconductor surface. The applied tip-sample bias is essentially screened by the surface states and may entirely drop in the insulating gap. In STS experiments the applied tip-sample bias can therefore be used as an energy scale to determine the energetic distribution of the local density of states. However, on semiconductors with a low surface state density, such as H-terminated $\mathrm{Si}$, cleaved $\mathrm{GaAs}(110)$ or the layered materials $\mathrm{WSe}_{2}$ and $\mathrm{WS}_{2}$, the situation is far more complex, since the applied tunneling voltage and the work function difference between the tip and the sample lead to an additional bandbending at the semiconductor surface and nonequilibrium effects need to be considered..$^{5-14}$

In a one-dimensional model the bandbending in a metalinsulator-semiconductor (MIS) diode was first determined by Garret and Brattain ${ }^{15}$ and Kingston and Neustadter ${ }^{16}$ by integrating the Poisson equation, including accumulation, depletion and inversion at the semiconductor surface. For a known bandbending the tunneling current can then be calculated using a tunneling theory for a MIS diode. ${ }^{5-10,14,17}$ The question whether this quasi-equilibrium approach is valid for

${ }^{\text {a)} E l e c t r o n i c ~ m a i l: m a t t h e s @ h m i . d e ~}$
STS measurements is still controversly discussed. ${ }^{5,10,12-14}$ It was first recognized by Feenstra and Stroscio ${ }^{5}$ that due to an extraction of the minority charge carriers, the semiconductor surface might not invert for the reverse bias situation. Bolotov et al. ${ }^{13}$ were able to measure this minority charge carrier extraction current on $\mathrm{H}$-terminated $\mathrm{Si}(111)$ samples. Depending on the temperature and the level of doping, they observed a different saturation level in the tunneling current for the reverse bias situation. For macroscopic MIS devices with a thin insulating gap, this is a well known effect. The numerical calculations of Green et al. ${ }^{28}$ showed, that in the case of a finite tunneling current, the minority charge carriers at the semiconductor surface may not be in thermal equilibrium with the charge carrier distribution in the bulk. As a result, the bandbending under reverse bias situation is not limited by the band gap anymore. For a $p$-type semiconductor this means, that a strong rectification in the $I-V$ curves will be observed, since for negative sample voltages tunneling of electrons from the valence band of the semiconductor into unoccupied states of the metal is not possible.

Additional insight into the influence of such nonequilibrium effects on the minority charge carrier distribution can be obtained by illumination of the tunneling junction. ${ }^{11-13,18-24}$ Using light with a photon energy of $h \nu$ $>E_{g}$, electron-hole pairs are created in the semiconductor. On semiconductors with a high surface state density it is essentially the balance between photoexcitation, diffusion and surface recombination of the minority charge carriers, which leads to a lateral shift of the $I-V$ curves under illumination, usually described by an additional local surface 
photovoltage (LSPV). ${ }^{11,19-21}$ The influence of tip- and biasinduced bandbending was analyzed by McEllistrem et al. ${ }^{11}$ who measured the voltage dependence of the LSPV for Si surfaces with different surface state distributions and densities. They showed that on H-terminated $\mathrm{Si}(111)$ the presence of the tunneling tip and the applied tip-sample bias significantly influences the bandbending at the semiconductor surface. However, the contribution of direct tunneling of photoexcited carriers, as observed by several other authors, ${ }^{12,13,22-24}$ was neglected in their electrostatic approach to calculate the LSPV. On the other hand, an interpretation of the observed $I-V$ curves under illumination by a simple superposition of dark current and voltage independent photocurrent, ${ }^{12,23}$ as generally used for the description of macroscopic solar cells, cannot explain the experimental results. ${ }^{12,13,22,24}$ Therefore, if one attempts to model the dependence of the photoinduced tunneling current (PITC) on the light intensity, the tip-sample distance and the tunneling voltage, a more detailed description is needed.

In this article, we present detailed STS measurements on the van der Waals surface of a $\mathrm{WS}_{2}$ single crystal. Due to the layered structure of this material, the (0001) van der Waals surface is free of intrinsic surface states in the forbidden band gap. We will show that on semiconductors with a low surface state density, the $I-V$ characteristics under dark condition and under illumination can both be reproduced by a one-dimensional MIS model. We discuss the measured dependence of the PITC on the tip-sample distance, the tunneling voltage and the light intensity in terms of analytical calculations. Non-equilibrium effects due to the finite tunneling current need to be considered in the calculation of the tipinduced bandbending, while surface recombination may be neglected in this approach.

\section{EXPERIMENT}

The STS measurements were performed on $p$-doped $\mathrm{WS}_{2}$ single crystals $\left(p=10^{17} \mathrm{~cm}^{-3}, E_{g}=1.3 \mathrm{eV}\right)$, which had been grown by chemical vapor transport. Clean, atomically flat (0001) surfaces were obtained by cleavage in air and immediate transfer into a high vacuum chamber $\left(10^{-6}\right.$ mbar). $I-V$ curves were measured using a homebuilt STM and mechanically cut PtIr tips. For photoexcitation we used the unfocused light of a HeNe laser $\left(633 \mathrm{~nm}, 500 \mathrm{~mW} / \mathrm{cm}^{2}\right)$.

In order to measure $I-V$ curves with and without illumination for the same tip-sample distance we used a special setup: The incident light is modulated by a mechanical chopper at $0.5 \mathrm{kHz}$. The $z$-feedback loop is active only during the first half of the dark phase and the tip-sample distance is adjusted for a given reference voltage $U_{\text {ref }}$ and reference current $I_{\text {ref }}$. Then the feedback loop is switched off and in the second half of the dark phase the tunneling current is measured for a fixed tunneling voltage $U_{t}$. With the feedback loop still inactive the light turns on and the tunneling current under illumination is measured at the same voltage $U_{t}$. Then the light turns off again and the feedback loop readjusts the tip-sample separation. By varying $U_{t}$ for a fixed value of $U_{\text {ref }}$ and $I_{\text {ref }}$ the $I-V$ curves are thus measured simultaneously

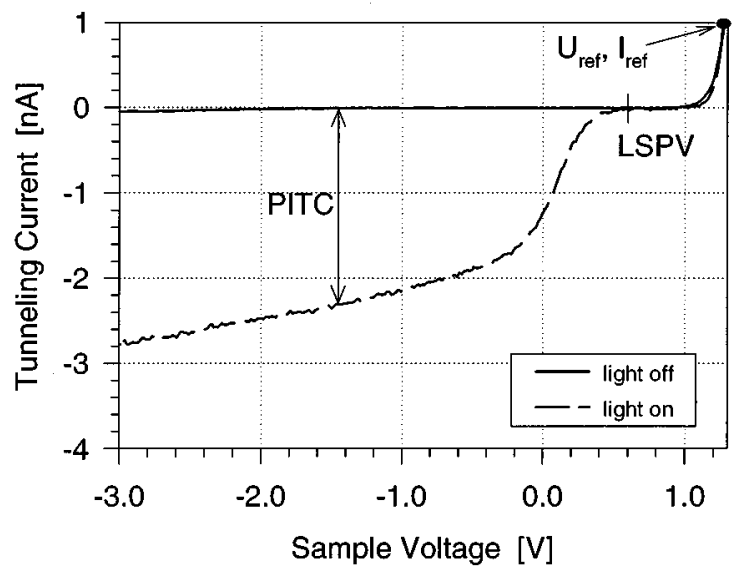

FIG. 1 . $I-V$ curves with and without illumination for a $p$-type $\mathrm{WS}_{2}$ sample. Reference sample voltage $U_{\text {ref }}=1.25 \mathrm{~V}$, reference current $I_{\text {ref }}=1 \mathrm{nA}$.

with and without illumination for the same tip-sample separation. Details of this interrupted feedback technique, which can be used for a lateral mapping of the PITC and the LSPV as well, is presented elsewhere. ${ }^{25}$

\section{EXPERIMENTAL RESULTS}

Figure 1 shows typical $I-V$ curves on a $p$-type $\mathrm{WS}_{2}$ sample. The tip-sample separation was adjusted under dark conditions for a reference voltage of $U_{\text {ref }}=1.25 \mathrm{~V}$ and a reference current $I_{\text {ref }}=1.0 \mathrm{nA}$.

The dark current increases exponentially for positive sample voltages, while for negative sample voltages up to $-3 \mathrm{~V}$ no tunneling current can be detected. Under illumination a photoinduced contribution to the tunneling current (PITC) is observed, which shows a strong dependence on the tunneling voltage. While for positive voltages $U_{t}>0.6 \mathrm{~V}$ the tunneling current is not influenced by the illumination, a strong increase of the PITC is observed for $0 \mathrm{~V}<U_{t}<0.6 \mathrm{~V}$. For more negative voltages, only a weak linear dependence of the PITC on the applied voltage is observed. The LSPV, which is defined as the voltage, where the tunneling current under illumination is zero, is about $0.6 \mathrm{~V}$. In the following sections the measured dependencies of the $I-V$ curves on various parameters are discussed in detail.

\section{A. Current-voltage curves under dark conditions}

In Fig. 2 the dark current for positive voltages is shown in a semi-logarithmic plot. In order to measure the $I-V$ curves for different tip-sample distances the reference voltage $U_{\text {ref }}$ was varied. The variation of the reference voltage in Fig. 2 was measured to change the tip-sample distance by about $0.8 \AA$. Due to the change in the tunneling transmission coefficient this results in a vertical shift of the $I-V$ curve in the semi-logarithmic plot. Similar to the measurements of Feenstra and Stroscio ${ }^{5}$ on $\operatorname{GaAs}(110)$ two contributions to the tunneling current can be identified. The first steep increase in the tunneling current for $U_{t}<1.2 \mathrm{~V}$ is attributed to the tunneling of electrons from the tip into dopant induced unoccupied states in the valence band $\left(J_{V}\right)$. The slope of the $I-V$ 


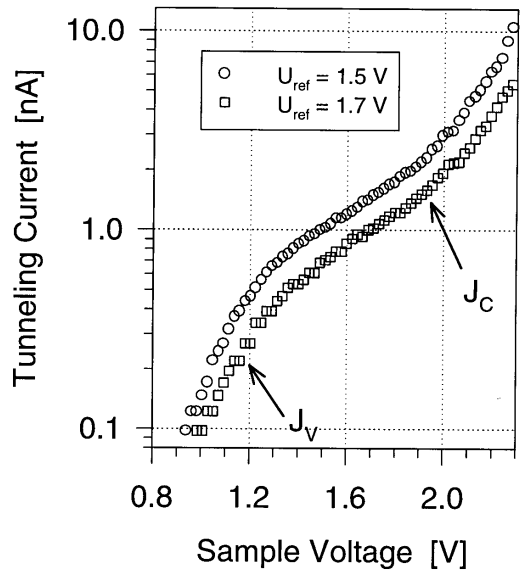

FIG. 2. $I-V$ curves without illumination for a $p$-type $\mathrm{WS}_{2}$ sample for two different reference voltages $\left(I_{\text {ref }}=1 \mathrm{nA}\right)$.

curve then decreases, while for larger tip-sample biases $\left(U_{t}>1.7 \mathrm{~V}\right)$ the second steep increase in the current is caused by the electrons tunneling from the tip into unoccupied states of the conduction band $\left(J_{C}\right)$.

\section{B. Current-voltage curves under illumination}

The dependence of the PITC on the reference voltage $U_{\text {ref }}$ is shown in Fig. 3. Varying the reference voltage by $0.5 \mathrm{~V}$ corresponds to a change of the tip-sample distance of about $2 \AA$.

For negative sample voltages $U_{t}<-0.8 \mathrm{~V}$ all the $I-V$ curves approach the same line, i.e., the PITC gets independent on the tip-sample separation. For sample voltages $-0.8 \mathrm{~V}<U_{t}<0.6 \mathrm{~V}$ a systematic variation of the PITC with the tip-sample distance is observed: For small tip-sample distances $\left(U_{\text {ref }}=1 \mathrm{~V}\right)$, the PITC increases strongly in the range $0.3 \mathrm{~V}<U_{t}<0.6 \mathrm{~V}$ and only a weak linear dependence on the sample voltage is observed for $U_{t}<0.3 \mathrm{~V}$. Increasing the

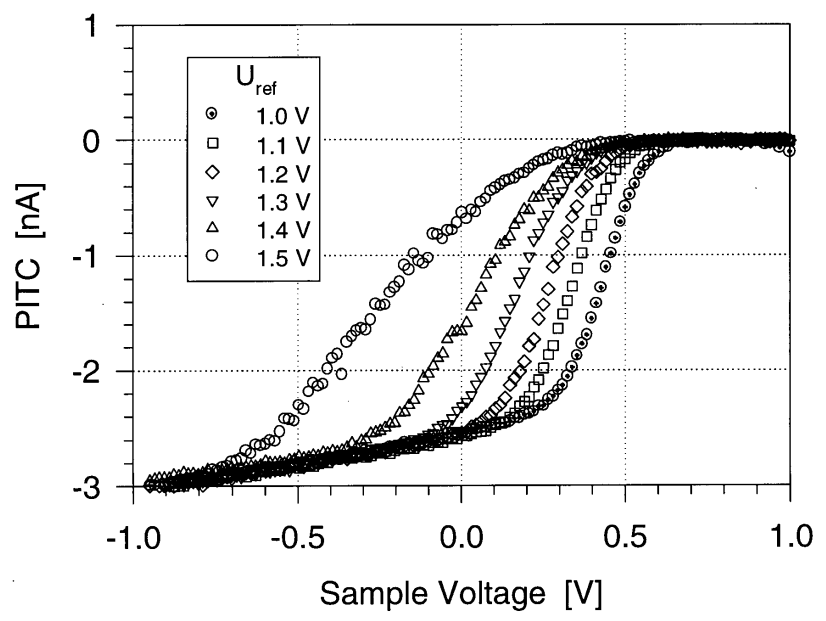

FIG. 3. PITC (additional tunneling current under illumination) for a $p$-type $\mathrm{WS}_{2}$ sample for different reference voltages corresponding to a variation of the tip-sample distance of about $2 \AA\left(I_{\text {ref }}=1 \mathrm{nA}\right)$.

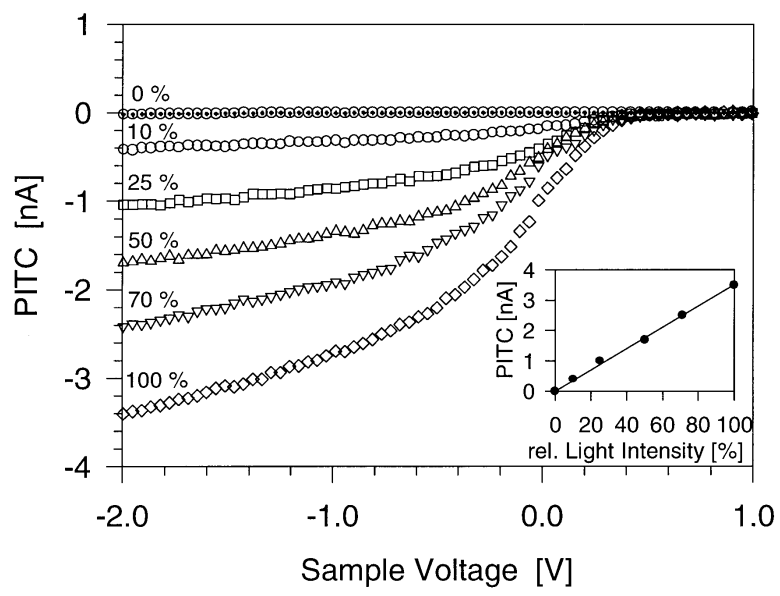

FIG. 4. PITC (additional tunneling current under illumination) for a $p$-type $\mathrm{WS}_{2}$ sample measured for different light intensities $\left(U_{\text {ref }}=1.3 \mathrm{~V}, I_{\text {ref }}=1\right.$ nA). $100 \%$ light intensity corresponds to $500 \mathrm{~mW} / \mathrm{cm}^{2}$. The inset shows the PITC at $U_{t}=-2 \mathrm{~V}$ and a linear fit as a function of laser intensity.

tip-sample distance ( $U_{\text {ref }} \approx 1.1 \mathrm{~V}$ ) leads to a small shift of the PITC curve to more negative sample voltages, while for larger tip-sample voltages $\left(U_{\text {ref }}>1.2 \mathrm{~V}\right)$ the increase of the PITC is stretched to more negative voltages. The LSPV shows no significant dependence on the tip-sample distance.

For a fixed tip-sample separation $\left(U_{\text {ref }}=1.3 \mathrm{~V}, I_{\text {ref }}=1\right.$ $\mathrm{nA}$ ), the PITC as a function of light intensity is shown in Fig. 4. For negative voltages the PITC is proportional to the light intensity. The inset shows the PITC and a linear fit at a sample voltage of $U_{t}=-2 \mathrm{~V}$. Again no significant variation of the LSPV can be measured.

\section{Variation of the tip-sample distance}

One striking difference of the $I-V$ curves measured under dark or illuminated conditions is the dependence of the tunneling current on the tip-sample distance. To investigate this

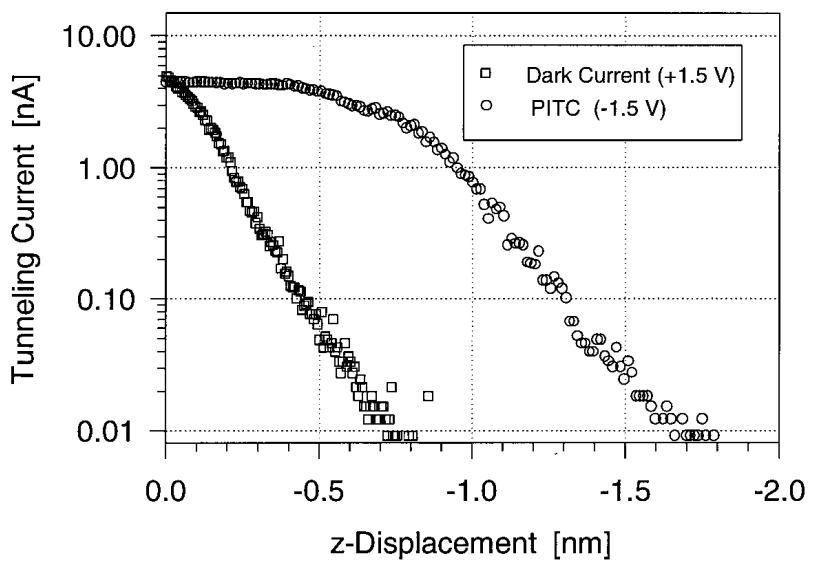

FIG. 5. Dependence of the dark current at $U_{t}=1.5 \mathrm{~V}$ and the PITC at $U_{t}$ $=-1.5 \mathrm{~V}$ on the tip-sample distance. The tip-sample distance was adjusted in the dark at $U_{\text {ref }}=1.5 \mathrm{~V}, I_{\text {ref }}=5 \mathrm{nA}$. During the measurement of the dark tunneling current at $U_{t}=1.5 \mathrm{~V}$ and the PITC at $U_{t}=-1.5 \mathrm{~V}$, an additional $z$-piezo voltage was applied to increase the tip-sample separation. 
effect in more detail, we explicitly varied the tip sample distance while measuring the dark current and the PITC simultaneously. To obtain the spectra shown in Fig. 5 the tipsample distance was adjusted in the dark for $U_{\text {ref }}$ $=1.5 \mathrm{~V}, I_{\text {ref }}=5 \mathrm{nA}$. Then an additional $z$-voltage was applied to retract the tip while measuring the dark current at $U_{t}=+1.5 \mathrm{~V}$ and the PITC at $U_{t}=-1.5 \mathrm{~V}$.

As expected, the dark current depends exponentially on the tip-sample distance and changes by one order of magnitude within a $z$-displacement of $\Delta z \approx 2 \AA$. On the other hand a variation of $\Delta z \approx 4 \AA$ does not change the PITC. A $z$-displacement of more than $5 \AA$ is necessary to reduce the PITC significantly. For more negative sample voltages $U_{t}$ $<-1.5 \mathrm{~V}$ even a larger $z$-displacement was necessary to observe changes in the PITC. As will be shown in the following section, this behavior is explained by the fact that at large reverse bias the junction behaves like a current source of the photogenerated minority carriers.

\section{THEORETICAL CONSIDERATIONS AND DISCUSSION}

An interpretation of the observed $I-V$ characteristics can be given on the base of a one-dimensional MIS model. Table I summarizes the parameters used in the calculations. Parameters were taken from the literature or were chosen to reflect the experimental conditions. For the layered semiconductor materials, such as $\mathrm{WS}_{2}$, a large variation in the measured electronic properties can be found in the literature, especially the anisotropy of the electronic transport properties is still controversly discussed. ${ }^{26}$ This is one reason why no attempt will be made to fit the calculated results quantitatively to the experimental data. However, the one-dimensional calculations can serve as a more general qualitative model for the interpretation of tunneling spectroscopy on semiconductors with a low surface state density.

\section{A. Current-voltage curves under dark conditions}

A calculation of the tunneling current under dark conditions is made by including a tunneling transmission coeffi-
TABLE I. Parameters used in the numerical calculations of $I-V$ curves for $p$-doped $\mathrm{WS}_{2}$ single crystals.

\begin{tabular}{lc}
\hline \hline Effective tunneling mass $m$ & $0.5 m_{0}$ \\
Band gap $^{\mathrm{a}} E_{g}$ & $1.34 \mathrm{eV}$ \\
Work function (tip) $\Phi_{t}$ & $5.0 \mathrm{eV}$ \\
Electron affinity $^{\mathrm{b}} \chi$ & $4.5 \mathrm{eV}$ \\
Dielectric constant $^{\mathrm{a}} \epsilon_{s}$ & $10 \epsilon_{0}$ \\
${\text { Intrinsic carrier concentration } n_{i}}^{14} \mathrm{~m}^{-3}$ \\
Minority carrier lifetime ${ }_{n}^{\mathrm{a}} \tau_{n}$ & $10^{\mathrm{a}} \times 10^{-6} \mathrm{~s}$ \\
Min. carrier diffusion length ${ }^{\mathrm{a}} L_{n}$ & $10^{-4} \mathrm{~m}$ \\
Absorption coefficient ${ }^{\mathrm{c}} \alpha$ & $10^{7} \mathrm{~m}^{-1}$ \\
Acceptor concentration $N_{A}$ & $10^{17} \mathrm{~cm}^{-3}$ \\
Effective photon flux $P_{L}$ & $10^{20} \mathrm{~cm}^{-2} \mathrm{~s}^{-1}$ \\
\hline \hline
\end{tabular}

${ }^{a}$ Reference 30.

${ }^{\mathrm{b}}$ Reference 32.

${ }^{\mathrm{c}}$ Reference 33 .

cient $T$ (WKB-approximation) into the theory of a metalsemiconductor contact. $^{27-29}$ The tunneling current contribution of the valence band $J_{V}$ is described by a thermionic emission current, ${ }^{27}$ while the tunneling current contribution of the conduction band $J_{C}$ is described by a minority carrier injection theory. ${ }^{28,29}$ This is a simplication compared to the method first presented by Feenstra and Stroscio, ${ }^{5}$ however, it is justified for low or moderate doping levels. In this case we expect thermally activated charge transfer processes to dominate over tunneling through the barrier inside the semiconductor.

To calculate $J_{V}$ and $J_{C}$ the bandbending as a function of the sample bias has to be known. Neglecting charges in surface states the potential drop in the tunneling gap can be calculated for a one-dimensional sheet capacitor as $U_{i}$ $={ }_{z} Q_{\mathrm{sc}} / \epsilon_{0}$, where $Q_{\mathrm{sc}}$ is the surface charge density due to the space charge region of the semiconductor. $Q_{\mathrm{sc}}$ is calculated as a function of the bandbending $e U_{d}$ by integrating Poisson's equation. ${ }^{15,16}$ Including accumulation, depletion and inversion at the semiconductor surface one gets

$$
Q_{s c}= \pm \sqrt{2 \epsilon_{s} k_{B} T N_{A}\left[\left(\exp \left(\frac{e U_{d}}{k_{B} T}\right)-\frac{e U_{d}}{k_{B} T}-1\right)+\frac{n_{i}^{2}}{N_{A}^{2}}\left(\exp \left(-\frac{e U_{d}}{k_{B} T}\right)+\frac{e U_{d}}{k_{B} T}-1\right)\right]}
$$

where the + sign corresponds to $U_{d}>0$, the - sign to $U_{d}$ $<0$. Varying $U_{d}$ in Eq. (1) and considering the work functions of the tip and the sample, the bandbending as a function of the tunneling voltage is obtained.

Figure 6 shows the bandbending $U_{d}$ and the resulting tunneling current as a function of the sample voltage $U_{t}$ for a tip-sample separation of $7 \AA$. The corresponding energy band diagrams are shown in Fig. 7.

The solid lines in Fig. 6 are obtained if the space charge density as described by Eq. (1) is used for the calculations. In
Fig. 6(a) three different slopes can be observed: Accumulation for $U_{t}>0.65 \mathrm{~V}$, depletion for $-0.7 \mathrm{~V}<U_{t}<0.65 \mathrm{~V}$ and inversion for $U_{t}<-0.7 \mathrm{~V}$ of the semiconductor surface can be distinguished. The tunneling current in Fig. 6(b) is obtained by adding the contribution of the valence band and the conduction band using the potential relations of Fig. 6(a).

For small positive voltages the tunneling current first increases due to the tunneling of electrons from the tip into unoccupied states of the $p$-type semiconductor valence band $\left(J_{V}\right.$ (maj.), Fig. $7($ b) $)$. This contribution reflects the voltage 

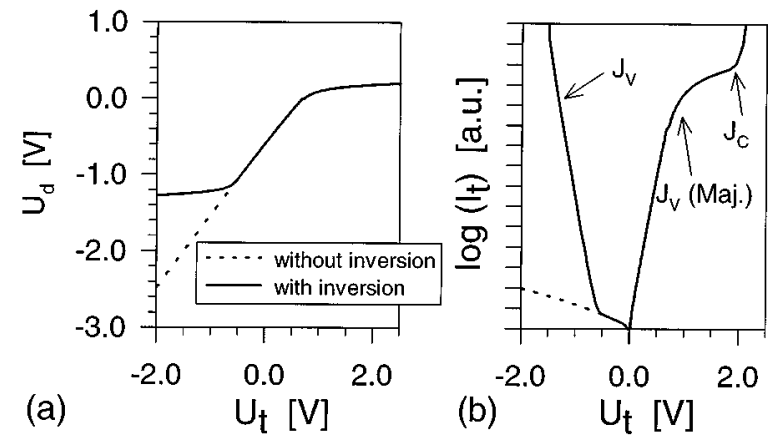

FIG. 6. (a) Bandbending as a function of the sample voltage for a tip-sample distance of $7 \AA$. Variation of the tip sample distance by $\pm 2 \AA$ does not significantly change the result. The solid line is obtained if the thermal inversion is considered (quasi-equilibrium approach). (b) Corresponding tunneling current. The arrows indicate the dominant contributions.

dependence of the bandbending, i.e., when the semiconductor gets into accumulation the slope in the $I-V$ curve is reduced. For larger positive voltages the additional applied voltage essentially drops in the tunneling gap and the Fermi level of the tip reaches the conduction band. The corresponding increase in the tunneling current is attributed to the tunneling of electrons from the tip into unoccupied states of the conduction band $\left(J_{C}\right.$, Fig. 7(a)).

The measured $I-V$ characteristics in Fig. 2 show the same behavior. The observed dependence of the tunneling current on the reference voltage is essentially caused by the tunneling transmission coefficients and bandbending effects. Therefore, without including a detailed density of state distribution within the semiconductor bands, the most significant features of the dark $I-V$ spectra for the forward bias (a)

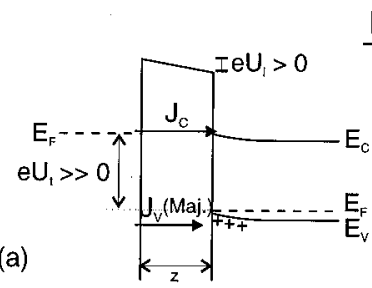

(c)

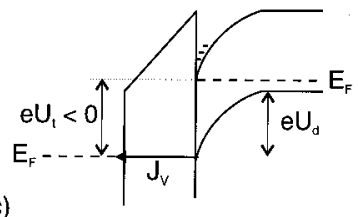

Light off:

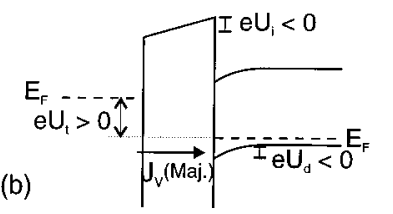

(d)

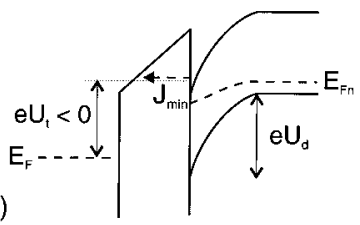

FIG. 7. Schematic view of the energy band diagrams and the tunneling currents (flow of electrons) for the $p$-type $\mathrm{WS}_{2}$ MIS diode under different bias conditions without illumination. Due to the assumed work function difference between the tip and the semiconductor a zero bias bandbending of $U_{d} \approx-0.60 \mathrm{~V}$ is obtained. (a) $U_{t} \gg 0$ : accumulation of majority carriers at the semiconductor surface; (b) $U_{t}>0$ : depletion; (c) $U_{t}<0$ : inversion; (d) Same as (c) neglecting the inversion charge. Due to a finite tunneling current $J_{\min }$ the quasi-Fermi level for the electrons $E_{F n}$ is not constant within the semiconductor.

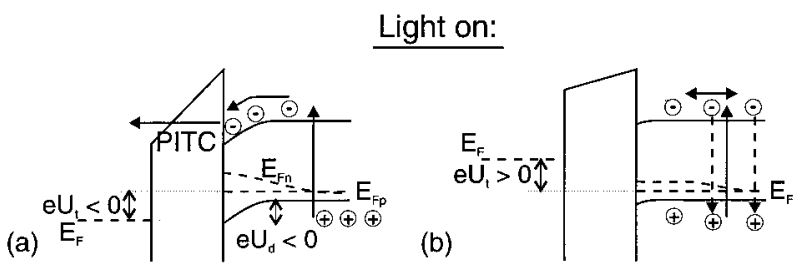

FIG. 8. Schematic view of the energy band diagrams and the tunneling currents for the $p$-type $\mathrm{WS}_{2}$ MIS diode under different bias conditions with illumination. (a) For negative sample voltages diffusion and separation of the photoexcited electron-hole pairs in the space charge region leads to a photoinduced tunneling current (PITC). (b) For positive sample voltages the bandbending at the semiconductor surface is reduced and back diffusion and bulk recombination of the photoexcited electron-hole pairs dominates.

situation can be well described by this simplified macroscopic theory.

For negative tunneling voltages (reverse bias) the tunneling current increases exponentially when the Fermi level of the tip reaches the valence band and tunneling of electrons from the semiconductor into the tip becomes possible $\left(J_{V}\right.$, Fig. 7(c)). However, in the dark $I-V$ curve in Fig. 1 no significant reverse current was measured. In the quasiequilibrium approach used for the calculation of the solid lines in Fig. 6 it was assumed that the electrostatic calculation of the bandbending is valid in the presence of a finite tunneling current. However, if the minority carrier generation rate is small compared to the tunneling rate no inversion layer at negative potentials exists. ${ }^{5,28}$ The dotted lines in Fig. 6 show the bandbending and the resulting tunneling current if the inversion at the semiconductor surface is neglected in Eq. (1). This assumption will be justified by the nonequilibrium calculations in the following section. For negative sample voltages, the bandbending is not limited by the band gap (Fig. 7(d)). Since in this case the Fermi level of the tip does not reach the valence band only a small tunneling current $J_{V}$ is observed at negative voltages. The minority carrier contribution $J_{\min }$ to the tunneling current is too small to be seen in Fig. 6 and the resulting $I-V$ curve shows the measured rectifying behavior.

\section{B. Current-voltage curves under illumination}

For the calculation of the photoinduced tunneling current a one-dimensional MIS solar cell theory similar to the one described by Srivastava and Bhatnagar ${ }^{30}$ and Abdou and Habib $^{31}$ will be used. Again, any contribution of surface states will be neglected. Since in our measurements no significant dark current was detected in the voltage range where a photoinduced signal was observed (Fig. 1), the contribution of the tunneling current from the valence band is neglected in this section. The energy band diagram and the current flow under illumination are schematically shown in Fig. 8.

Illumination of a p-type semiconductor creates free electrons in the conduction band. For a negative bandbending $U_{d}<0$ the contribution of the minority carriers in the con- 
duction band to the tunneling current density $J_{\min }$ is now given by $^{31}$

$$
J_{\min }=-e P_{L}+e \frac{L_{n}}{\tau_{n}}\left(n(w)-n_{p 0}\right) .
$$

The first term describes the current if all the electron-hole pairs are collected within the diffusion length $L_{n}$ and are separated in the space charge region of the semiconductor. $P_{L}$ is the effective photon flux, which, in a one-dimensional theory with a high optical absorption coefficient $\left(\alpha^{-1} \ll L_{n}\right)$, is proportional to the absorbed photon flux. The second term in Eq. (2) is the back diffusion current, which represents the photoexcited carriers that are lost by back diffusion and recombination in the bulk.

Using Maxwell-Boltzmann statistics the minority carrier concentration $n(w)$ at the edge of the depletion region can be described as $n(w)=n(0) \exp \left(e U_{d} / k_{B} T\right)$, where $n(0)$ is the minority carrier concentration at the semiconductor surface. Splitting $n(0)$ into an equilibrium concentration for zero bias without illumination and a non-equilibrium concentration $n_{e}$ of excess carriers at the surface with applied bias and illumination gives

$$
n(w)=n_{p 0} \exp \left(\frac{e \Delta U_{d}}{k_{B} T}\right)+n_{e} \exp \left(\frac{e U_{d}}{k_{B} T}\right),
$$

where $n_{p 0}$ is the bulk minority carrier concentration and $\Delta U_{d}$ is the change in the bandbending due to the applied bias and the illumination.

On the other hand, the excess carriers $n_{e}$ will give a tunneling current density from the conduction band into unoccupied states of the metal

$$
J_{\min }=-e v_{n} T_{n} n_{e},
$$

where $v_{n}=\sqrt{\left(k_{B} T / 2 \pi m\right)}$ is the effective thermal velocity of the minority carriers and $T_{n}$ the tunneling transmission coefficient at the conduction band edge. ${ }^{30}$ Due to the continuity equation of charge carriers at the surface, Eqs. (2) and (4) have to be identical. This gives the excess charge as

$$
n_{e}=\frac{P_{L}-n_{p 0} \frac{L_{n}}{\tau_{n}}\left(\exp \left(\frac{e \Delta U_{d}}{k_{B} T}\right)-1\right)}{v_{n} T_{n}+\frac{L_{n}}{\tau_{n}} \exp \left(\frac{e U_{d}}{k_{B} T}\right)} .
$$

The first term in the nominator describes the excess charge due to the illumination of the semiconductor, while the second term takes the thermally generated minority carriers into account. This excess charge of minority carriers has to be considered in the calculation of space charge density for the potential drop $U_{i}:^{31}$

$$
Q_{\mathrm{sc}} \rightarrow Q_{\mathrm{sc}}^{*}-\sqrt{2 \epsilon_{s} k_{B} T_{n} n_{e}},
$$

where $Q_{\mathrm{sc}}^{*}$ is the carrier concentration from Eq. (1) neglecting the inversion due to the applied bias, since this inversion now is included in the self-consistent calculation of the excess carrier concentration $n_{e}$. For a constant transmission coefficient $T_{n}$, the space charge $Q_{\mathrm{sc}}$ is only a function of the
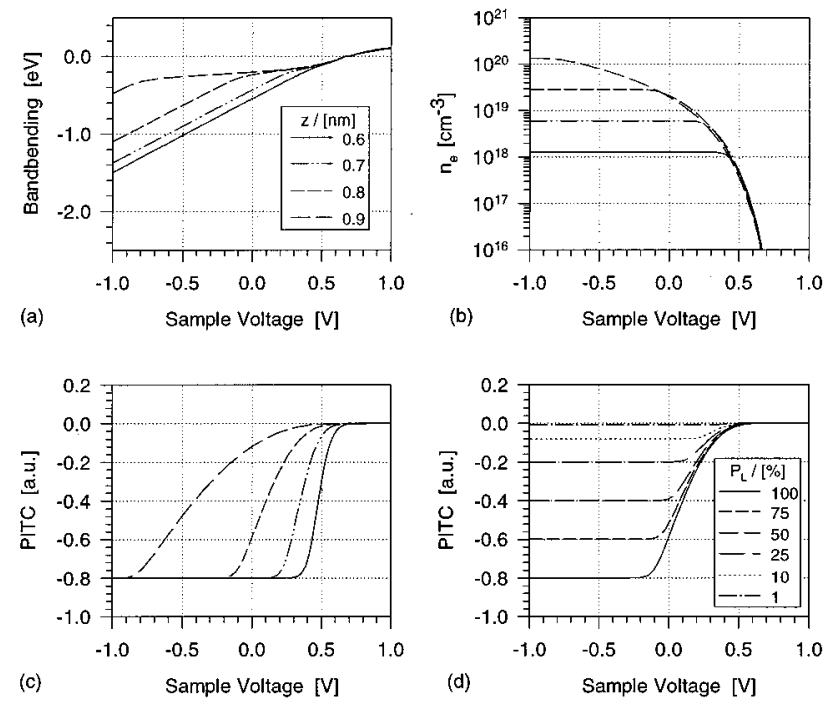

FIG. 9. Calculation of the photoinduced tunneling current. (a) Bandbending as a function of the applied tunneling voltage for different tip-sample distances and an effective photon flux of $P_{L}=10^{20} \mathrm{~cm}^{-2} \mathrm{~s}^{-1}$. (b) Corresponding excess carrier concentration $n_{e}$. (c) Resulting photoinduced tunneling current (PITC). (d) Variation of the PITC with the light intensity for a fixed tip-sample distance at $z=8 \AA$.

bandbending $U_{d}$ and the calculation of the bandbending as a function of the applied tunneling voltage is done as described before. Using Eqs. (4) and (5) the tunneling current from the conduction band is then obtained as a function of the tunneling voltage. For the light intensities and the doping concentration used in our experiments this calculations show, that the second term in the nominator of Eq. (5) can be neglected. This explains why the tunneling current of minority carriers $J_{\min }$ under dark conditions is too small to be measured by the STM. In addition, by setting $P_{L}=0$ this model can be used to study non-equilibrium effects of the minority charge carriers under dark conditions. For the parameters used in our calculations, this justifies the assumption that the inversion charge can be neglected in the calculation of the tip-induced bandbending. However, under illumination the minority charge carrier generation rate is drastically increased and the tunneling current $J_{\min }$ is equal to the PITC.

The bandbending and the corresponding excess carrier concentrations are shown in Figs. 9(a) and 9(b) for an effective photon flux $P_{L}=10^{20} \mathrm{~cm}^{-2} \mathrm{~s}^{-1}$. This value is chosen to be large compared to the light intensities used in our experiments, because experimental photoinduced tunneling currents of about $1 \mathrm{nA}$ show, that a lateral collection of photoexcited carriers from an area of several $\mu \mathrm{m}^{2}$ have to be considered.

For negative voltages $U_{t}<-0.8 \mathrm{~V}$ (Fig. 8(a)), the second term in the denominator of Eq. (5) (back-diffusion current) can be neglected. Figure 9(b) shows, that for decreasing voltages $n_{e}$ reaches a saturation level. This saturation level increases exponentially with increasing tunneling gap. For more positive voltages (Fig. 8(b)), the bandbending is reduced and due to the back-diffusion current the excess carrier concentration $n_{e}$ decreases rapidly. For a larger tip- 
sample distance $z$, this decrease in $n_{e}$ is stretched to more negative voltages. Figure 9(a) shows, that this is due to a reduction of the bandbending at the semiconductor surface for increasing tunneling gap. This reduced bandbending is the result of an accumulation of the photoexcited minority carriers at the semiconductor surface. Without illumination, no significant dependence of the bandbending on the different tip-sample separations would be seen in Fig. 9(a).

The resulting PITC is shown in Fig. 9(c). For negative sample voltages, the tunneling current reaches a saturation current, which is independent of the tip-sample distance (semiconductor limited) and proportional to the light intensity (Fig. 9(d)). For more positive voltages, the two terms in the denominator of Eq. (5) become comparable and a transition from a semiconductor limited to tunneling limited tunneling current occurs. The voltage dependence of this transition on the tip-sample distance is due to two effects: First, increasing the tip-sample distance leads to a smaller transmission coefficient $T_{n}$ in Eq. (5) and the second term in the denominator dominates at smaller voltages. Second, for larger tip-sample distances an optically induced inversion layer is produced at the semiconductor surface and the resulting photovoltage (reduction in the bandbending) stretches the transition to more negative voltages. Figure $9(\mathrm{~d})$ shows that for the same reason the saturation level in the PITC for lower light intensities is reached for more positive sample voltages.

All these effects are clearly observed in the measured PITC curves in Fig. 3 and Fig. 4. This shows that direct tunneling of photoexcited carriers is responsible for the observed photoinduced signals. For negative sample voltages, the PITC is semiconductor limited. This explains the weak dependence of the PITC on variations of the tip-sample distance below $5 \AA$ as observed in Fig. 5. These calculations show further, that the PITC is drastically reduced for $U_{d} \rightarrow 0$. This means that the LSPV is directly related to the flat band voltage of the semiconductor $\left(U_{t}\left(U_{d}=0\right)\right)$. If the dark current at this voltage is vanishingly small, no significant dependence of the LSPV on the tip-sample distance and the light intensity will be observed.

For $U_{d} \ll 0$, the PITC in Figs. 9(c) and 9(d) becomes independent of the sample voltage, while the measurements show a linear dependence of the PITC on the sample voltage. In the one-dimensional model the saturation of the PITC for negative voltages is due to the high absorption coefficient $\left(\alpha^{-1}<L_{n}\right)$ and the neglecting of recombination in the space charge region and at the surface. The finite slope of the PITC curve could be explained in a more detailed threedimensional model, where lateral collection of photoexcited carriers, surface recombination and spreading resistance effects would have to be considered.

\section{SUMMARY AND CONCLUSIONS}

In summary, we have performed tunneling spectroscopy with and without illumination on a $p$-type $\mathrm{WS}_{2}$ sample as a model system for semiconductors with a low surface state density. Using an interrupted feedback technique, $I-V$ curves under dark conditions and under illumination were obtained simultaneously for the same tip-sample separation. A qualitative interpretation was given using a onedimensional MIS model.

Without illumination the $I-V$ characteristics showed the typical rectifying behavior of a MIS diode with a thin insulating gap. For positive sample voltages, two voltage dependent current contributions were identified as tunneling into unoccupied states of the valence band and the conduction band. For negative sample voltages, no tunneling current could be detected. The rectifying behavior was explained by the absence of thermal equilibrium between the minority charge carrier concentration at the semiconductor surface and the bulk concentration.

These non-equilibrium effects are more drastically revealed in the $I-V$ curves measured under illumination for different tip-sample separations and different light intensities. For the illuminated surface, the surface excess charge and the tip-induced bandbending were obtained in a selfconsistent MIS model, where the continuity equation for the photogenerated minority carriers is taken into account. In spite of the fact that recombination in the space charge region or through surface states was neglected, the dependence of the PITC on the sample voltage, the tip-sample separation and the light intensity was reproduced by this onedimensional model. This shows in contrast to the interpretations of the measurements on H-terminated Si samples ${ }^{11}$ that direct tunneling of photoexcited carriers is the dominant process for a semiconductor with a low surface state density. The voltage dependence of the PITC was explained by a transition from direct tunneling of the photoexcited carriers to back-diffusion and recombination in the semiconductor bulk. A detailed analysis of the dependence of the PITC on the tip-sample separation and the light intensity shows, that for increasing tip-sample separation this transition is triggered by an optically induced accumulation of minority charge carriers. At the same time our non-equilibrium approach shows that under dark conditions the direct tunneling of minority charge carriers under reverse bias effectively extracts these charge carriers and does not allow an accumulation of minorities at the surface. Therefore an inversion cannot occur, which had been generally assumed in other simulations of $I-V$ spectra.

In spite of the fact that no special band structure of the valence and conduction band was used in our calculations, the general shape of the $I-V$ characteristics for dark and illuminated conditions could be reproduced. This indicates, that in the case of semiconductors with a low surface state density, the bulk properties, such as level of doping, minority generation rate, lifetime and diffusion length essentially determine the overall shape of the $I-V$ spectra. It should be emphasized that on semiconductor surfaces, which are not completely dominated by surface states, the standard interpretation of tunneling spectroscopy, which correlates the normalized differential conductivity to the energetic distribution of the local state density, may lead to significant misinterpretations. It definitely fails on surfaces, where surface 
states are absent or do not hinder tip-induced bandbending effects.

In addition, the influence of the tip on the charge carrier distribution inside the semiconductor is not limited to electrostatic interactions, but also includes non-equilibrium effects due to the finite tunneling current. However, our approach also shows that this opens the way to investigate semiconductor bulk properties with high lateral resolution if numerical methods can be elaborated, which may describe the problem in its three-dimensional nature.

${ }^{1}$ R. S. Becker, J. A. Golovchenko, D. R. Hamann, and B. S. Swartzentruber, Phys. Rev. Lett. 55, 2032 (1985).

${ }^{2}$ R. J. Hamers, R. M. Tromp, and J. E. Demuth, Phys. Rev. Lett. 56, 1972 (1986).

${ }^{3}$ R. M. Feenstra, W. A. Thompson, and A. P. Fein, Phys. Rev. Lett. 56, 608 (1986).

${ }^{4}$ J. A. Stroscio, R. M. Feenstra, and A. P. Fein, Phys. Rev. Lett. 57, 2579 (1986).

${ }^{5}$ R. M. Feenstra and J. A. Stroscio, J. Vac. Sci. Technol. B 5, 923 (1987).

${ }^{6}$ L. D. Bell, W. J. Kaiser, M. H. Hecht, and F. J. Grunthaner, Appl. Phys. Lett. 52, 278 (1988).

${ }^{7}$ M. Weimer, J. Kramar, and J. D. Baldeschwieler, Phys. Rev. B 39, 5572 (1989).

${ }^{8}$ J. Jahanmir, P. E. West, A. Young, and T. N. Rhodin, J. Vac. Sci. Technol. A 7, 2741 (1989).

${ }^{9}$ M. B. Johnson and J.-M. Halbout, J. Vac. Sci. Technol. B 10, 508 (1992).

${ }^{10}$ R. Maboudian, K. Pond, V. Bressler-Hill, M. Wassermeier, P. M. Petroff, G. A. D. Briggs, and W. H. Weinberg, Surf. Sci. Lett. 275, L662 (1992).

${ }^{11}$ M. McEllistrem, G. Haase, D. Chen, and R. J. Hamers, Phys. Rev. Lett. 70, 2471 (1993).

${ }^{12}$ F. R. F. Fan and A. J. Bard, J. Phys. Chem. 97, 1431 (1993).

${ }^{13}$ L. N. Bolotov, I. V. Makarenko, A. F. Shulekin, and A. N. Titkov, Surf. Sci. 331, 468 (1995).
${ }^{14}$ R. M. Silver, J. A. Dagata, and W. Tseng, J. Appl. Phys. 76, 5122 (1994).

${ }^{15}$ C. G. B. Garrett and W. H. Brattain, Phys. Rev. 99, 376 (1955).

${ }^{16}$ R. H. Kingston and S. F. Neustadter, J. Appl. Phys. 26, 718 (1995).

${ }^{17}$ R. Chapman, M. Kellam, S. Goodwin-Johansson, J. Russ, and G.-E. McGuire, J. Vac. Sci. Technol. B 10, 502 (1992).

${ }^{18}$ G. F. A. van de Walle, H. van Kempen, and P. Wyder, Appl. Phys. Lett. 50, 22 (1987).

${ }^{19}$ R. J. Hamers and K. Market, Phys. Rev. Lett. 64, 1051 (1990).

${ }^{20}$ Y. Kuk, R. S. Becker, P. J. Silverman, and G. P. Kochanski, Phys. Rev. Lett. 65, 456 (1990).

${ }^{21}$ D. G. Cahill and R. J. Hamers, J. Vac. Sci. Technol. B 9, 564 (1991).

${ }^{22}$ S. Akari, M.Ch. Lux-Steiner, M. Vögl, M. Stachel, and K. Dransfeld, J. Vac. Sci. Technol. B 9, 561 (1991).

${ }^{23}$ B. W. Wessels and L. Q. Qian, J. Vac. Sci. Technol. B 10, 1803 (1992).

${ }^{24}$ M. W. J. Prins, M. C. M. M. van der Wielen, R. Jansen, D. L. Abraham, and H. van Kempen, Appl. Phys. Lett. 64, 1207 (1994).

${ }^{25}$ Th. W. Matthes, Ch. Sommerhalter, A. Rettenberger, M. Böhmisch, J. Boneberg, M. Ch. Lux-Steiner, and P. Leiderer, Proceedings of the ICFSI-6, Cardiff, Wales, 1997 (to be published in Appl. Surf. Sci.).

${ }^{26} \mathrm{E}$. Bucher, in Photoelectrochemistry and Photovoltaics of Layered Semiconductors, edited by A. Aruchamy (Kluwer, Dordrecht, Netherlands, 1992).

${ }^{27}$ C.-Y. Wu, J. Appl. Phys. 51, 3786 (1980).

${ }^{28}$ M. A. Green, F. D. King, and J. Shewchun, Solid-State Electron. 17, 551 (1974).

${ }^{29}$ S.M. Sze, Physics of Semiconductor Devices, 2nd ed. (Wiley, New York, 1991).

${ }^{30}$ G. P. Srivastava and P. K. Bhatnagar, Solid-State Electron. 22, 581 (1979).

${ }^{31}$ A. A. Abdou and S. E.-D. Habib, Solid-State Electron. 29, 751 (1985).

${ }^{32} \mathrm{~W}$. Jaegermann, in Photoelectrochemistry and Photovoltaics of Layered Semiconductors, edited by A. Aruchamy (Kluwer, Dordrecht, Netherlands, 1992).

${ }^{33}$ A. R. Beal, J. C. Knights, and W. Y. Lang, J. Phys. C 5, 3540 (1972). 